\title{
La Universidad de El Salvador como espacio de reproducción de la violencia de género
}

\author{
La Universidad de El Salvador (University of El Salvador) \\ as a space for the reproduction of gender violence
}

ISSN 2071-8748

E-ISSN 2218-3345

\section{(c) $\$(0)$ BY NC SA}

URI: http://hdl.handle.net/11298/879

DOI: http://dx.doi.org/10.5377/entorno.v0i66.6727

\author{
María Ángela Rodríguez \\ Coordinadora de la Red de Investigación \\ maria.rodriguez5@ues.edu.sv \\ ORCID: 0000-0001-8748-6864 \\ Recibido: 21/08/18 \\ Aprobado:12/12/18
}

\section{Resumen}

El objetivo general de la investigación fue caracterizar las expresiones de violencia que se reproducen en el espacio universitario. El estudio es descriptivo, con enfoque mixto, se usó la técnica de la encuesta, aplicada a un total de 655 personas, a través de un muestreo estratificado aplicado en todas las facultades del campus central y de la Facultad Multidisciplinaria de Oriente. Además, se realizaron talleres, grupos focales y entrevistas con autoridades, docentes, personal administrativo estudiantes, sindicatos, gremios y asociaciones estudiantiles, entre febrero y abril de 2018.

Los resultados arrojaron que se reproducen todos los tipos de violencia, pero son la psicológica y emocional, y la sexual las más frecuentes, $65.75 \%$ y $15.53 \%$, respectivamente. Los espacios donde mayormente se

\section{Abstract}

The general objective of the research was to typify the expressions of violence that are reproduced in the spaces of the university. This is a descriptive study, with an integrated approach; the survey technique was utilize and administer to 655 people, through a stratified sampling applied in all the faculties of the main campus and from the eastern multidisciplinary faculty. In addition, workshops, focus groups and interviews were held with authorities, teachers, administrative staff, students, unions and student associations, between February and aril 2018. The results showed that all types of violence are reproduced, but the psychological and emotional, and sexual violence are the most frequent with 65,75 and $15,53 \%$ respectively. The spaces where violence is mostly reproduced are the classrooms, 41,69\%; the cubicles, $14,58 \%$, and the offices with $10,02 \%$. The indifference

1 Docente e investigadora de la Facultad de Ciencias Económicas de la Universidad de El Salvador. Coordinadora de la Red de Investigación en diferenciales de Género de Iberoamérica, filial Universidad de El Salvador. Además integran la Red Docentes de la Facultad de Jurisprudencia y Ciencias Sociales, Diana Merino, Multidisciplinaria Oriental, Azucena Retana y el Centro de Estudios de Género, Roselia Núnez. 
reproduce la violencia son las aulas, $41.69 \%$; cubículos, $14.58 \%$, y oficinas, un $10.02 \%$. Contribuyen a esta situación la indiferencia de la comunidad universitaria, los espacios solitarios y el desempeño de la seguridad del personal universitario, como los más determinantes.

Las mujeres son las más violentadas, $64.82 \%$; así como la población LGBTI, con $34.66 \%$, y los hombres en $18.52 \%$. Quienes más reproducen dicha violencia, según la opinión de más del $55 \%$ de la consulta, son los hombres de todos los sectores. Señalan que esto se debe a las relaciones de poder, aunado al desconocimiento de derechos por parte de la comunidad universitaria, y el miedo, entre otras.

Es innegable que la institución no escapa a los sistemas heteropatriarcal y machista. Pese a la vigencia de un marco normativo sobre los derechos de las mujeres, no se tiene mayor conocimiento del mismo, como tampoco de las instancias internas responsables de la implementación de la legislación. Todo ello contribuye a la falta de denuncia, que también es inducida por el temor y por la poca confianza en los procesos.

\section{Palabras clave}

Violencia contra la mujer; Delitos contra la mujer; Estereotipo (psicología); Agresividad (psicología). of the university community, the solitary spaces and the performance of the university security team contributes to this situation, as the most determining factor of this situation. Women are most commonly victims of violent aggression with a $64,82 \%$, followed by the population of lesbians, gays, bisexual, transsexuals, and intersexual with a $34,66 \%$; and men with a $18,52 \%$; Those who reproduce this violence in a major scale, according to the opinion of $55 \%$ of the interviewees, are men.

It is pointed out that this situation is due to the power relations, fear and others, all this join with the lack of knowledge of rights among the university community. It is undeniable that the institution does not escape from the male oriented and heteropatriarchal systems. Despite the validity, of a normative framework on women's rights, not enough is known, neither of the internal instances responsible for the implementation of the legislation. All this contributes to the lack of complaint, which is also induced by fear and by the little confidence in the processes.

\section{Keywords}

Violence against women, Crimes against women, Stereotype (psychology), Aggressiveness (psychology).

\section{Introducción}

La investigación se desarrolló en el marco de la Red de Investigación en Diferenciales de Género en la Educación Superior Iberoamericana, a la que se sumó la Universidad de El Salvador (UES) ${ }^{2}$ en 2017. El objetivo general fue caracterizar las expresiones de violencia que se reproducen en el espacio universitario de manera específica; se buscó identificar los tipos y expresiones de violencia, los espacios donde se expresa la violencia de género, así como los agentes que reproducen dicha violencia dentro de la universidad.
La Ley Especial Integral para una Vida Libre de Violencia contra las Mujeres [LEIV] (Asamble Legislativa, 2011) tiene como objeto, en su artículo 1, establecer, reconocer y garantizar el derecho de las mujeres a una vida libre de violencia, indicando que para ello deben asegurarse esos derechos mediante la elaboración de políticas públicas encaminadas a detectar, prevenir, atender, proteger, reparar y sancionar la violencia contra ellas.

Formalmente, en la UES se cuenta con marcos normativos a favor de las mujeres, como son la Política de Equidad de Género, el Primer Plan de Igualdad de Género $^{3}$ y el

2 Es la única universidad pública de El Salvador, fundada el 16 de febrero de 1841. A la fecha, tiene cuatro campus que albergan a 12 facultades, 9 de ellas en el campus central. ${ }^{4}$ Estudiante de la licenciatura en antropología de la Universidad Tecnológica de El Salvador.

3 Desde el 24 de mayo de 2007, la UES a propuesta del Centro de Estudios de Género de la Universidad de El Salvador CEG, cuenta con la Política de Equidad Género de la Universidad de El Salvador, aprobada por acuerdo No. 064-2005-2007 (VII-2) del Consejo Superior Universitario, y por Acuerdo No. 245-A de Rectoría de la Universidad de El Salvador, el 9 de abril de dos mil catorce se aprobó el primer Plan de Igualdad. 
Reglamento Disciplinario (Asamblea General Universitaria, 2001). Este último, en su artículo 11, literal " $p$ ", contempla como infracción grave la figura del acoso sexual, entendido como "las conductas inequívocas tendientes a obtener una relación sexual, manifestada en acechos o tocamientos rechazados por la otra persona, o promesas de promoción de una asignatura, o la obtención de un beneficio académico o administrativo a cambio de la concesión de un favor sexual". Sin embargo, estos instrumentos no han sido actualizados de acuerdo con la normativa nacional vigente, para que cumplan su cometido.

Si bien en los tesarios y archivos de investigación de la universidad hay documentos relacionados con el tema de género, hasta el momento no se encontró ninguno referido al análisis de la reproducción de la violencia de género en el campus; esto aun cuando hay voces que gritan silenciosamente que en sus espacios se dan diferentes expresiones de violencia. De ahí que la Red de Investigadoras de la UES identificó la necesidad de realizar una investigación sobre la reproducción de la violencia de género, con el fin de producir datos fiables a partir de los cuales las autoridades puedan tomar medidas para evitar que en la institución se reproduzca dicha violencia.

Se identificaron y analizaron los tipos y expresiones de violencia de género que se reproducen dentro de la UES, así como los espacios y agentes reproductores de la misma. Las técnicas utilizadas permitieron, por el lado cualitativo conocer la percepción de representantes de los diferentes sectores que conforman la comunidad universitaria: docente; administrativo y servicios, estudiantil y autoridades; mientras que la información cuantitativa reforzó dichas percepciones. En conjunto, este conocimiento ha permitido delinear una propuesta para el abordaje de la problemática de violencia dentro de la universidad presentada a las autoridades centrales.

En resumen, la investigación busca contribuir al logro de la igualdad y la erradicación de la violencia contra las mujeres mediante propuestas que fomenten una cultura de igualdad y respeto, con la esperanza de servir de ejemplo para el resto de las instituciones de educación superior del país y la sociedad salvadoreña.

\section{Metodología}

El tipo de investigación es descriptiva, con enfoque mixto. Se buscó información de carácter cualitativo como cuantitativo; se utilizó la inducción y la deducción de acuerdo con la información disponible.

Como unidades de análisis se definió a estudiantes, docentes, autoridades y personal administrativo y de servicios.

En la recopilación de información de carácter cualitativa se usaron diferentes técnicas, entre ellas: entrevistas en profundidad dirigidas a personas de mayor jerarquía en la institución, considerando su poder decisorio en la dinámica de la universidad, entre ellas el rector, vicerrector académico, las decanaturas, la presidencia y vicepresidencia de la Asamblea General Universitaria (AGU), Fiscalía y Defensoría de los Derechos Universitarios (DDU). Se incluyó como informantes a dirigentes sindicales del sector administrativo y de servicio, así como a representantes de las asociaciones y los gremios estudiantiles. En este rango, se entrevistó a 22 informantes. Además, se desarrollaron dos grupos focales con participación de personal administrativo del campus central; se trabajó con grupos de mujeres y hombres por separado. También se realizaron dos talleres con estudiantes, hombres y mujeres, del campus central, quienes compartieron sus percepciones y vivencias sobre las expresiones, tipos, agentes y espacios en los que se reproduce la violencia de género.

En el levantamiento de información de carácter cuantitativo, se utilizó la técnica de la encuesta, mediante un cuestionario estructurado en cuatro secciones. Cada una incluyó un listado de preguntas que permitieron cuantificar los tipos, expresiones, agentes y espacios de reproducción de la violencia de género en la Unidad Central y en la Facultad Multidisciplinaria Oriental (FMO), además de explorar las propuestas que desde la comunidad universitaria deben impulsarse para evitar la violencia de género.

El ámbito o universo del estudio fueron mujeres y hombres del campus central de la Universidad y la FMO, según se detalla en la tabla 1. 
Tabla 1. Población por sector. 2017

\begin{tabular}{|l|r|r|r|}
\hline \multirow{2}{*}{ Sector } & \multicolumn{3}{|c|}{ Cantidad } \\
\cline { 2 - 4 } & \multicolumn{1}{|c|}{ Mujeres } & \multicolumn{1}{|c|}{ Tombres } \\
\hline Estudiantes & 25,131 & 20,977 & $\mathbf{4 6 , 1 0 8}$ \\
\hline Personal docente & 612 & 1,277 & $\mathbf{1 , 8 8 9}$ \\
\hline Sector administrativo y de servicios & 433 & 268 & $\mathbf{7 0 1}$ \\
\hline Jefaturas & 15 & 40 & $\mathbf{5 5}$ \\
\hline Asamblea General Universitaria & 22 & 34 & $\mathbf{5 6}$ \\
\hline Total del universo & $\mathbf{2 6 , 2 1 3}$ & $\mathbf{2 2 , 5 9 6}$ & $\mathbf{4 8 , 8 0 9}$ \\
\hline
\end{tabular}

Fuente: elaboración propia en base a datos de la Administración Académica (Adacad) y la Unidad Técnica de Evaluación de la Universidad de El Salvador (Coteues), 2017.

Se utilizó la técnica del muestreo estratificado, con un error muestral de $3 \%$ y una confianza de $98 \%$. En el diseño de la muestra se consideraron proporciones de acuerdo con el tamaño de las Facultades y con la distribución por sexos, obteniendo un valor mínimo representativo de 287 personas, sin embargo, se aplicó el cuestionario a un total de 655, distribuidos en todos los sectores, según la tabla 2.

Tabla 2. Total de cuestionarios aplicados

\begin{tabular}{|c|c|c|c|}
\hline \multirow[t]{2}{*}{ Sector } & \multicolumn{3}{|c|}{$\begin{array}{l}\text { Incluye facultades del campus central y la Facultad de } \\
\text { Oriente }\end{array}$} \\
\hline & Masculino & Femenino & Total \\
\hline Estudiantes & 129 & 151 & 280 \\
\hline Docentes & 153 & 64 & 217 \\
\hline \multicolumn{4}{|c|}{ Personal administrativo y de servicios } \\
\hline Administrativo y servicios & 30 & 50 & 80 \\
\hline Jefaturas & 21 & 18 & 39 \\
\hline \multicolumn{4}{|c|}{ Asamblea General Universitaria } \\
\hline Representante propietario & 20 & 19 & 39 \\
\hline Total & 353 & 302 & 655 \\
\hline
\end{tabular}

Fuente: elaboración propia.

4 Para el período 2017-2019, no todas las facultades han elegido al total de representantes. 


\section{Resultados}

Se presentan los principales hallazgos del estudio sobre la reproducción de la violencia de género en la UES, a partir de la identificación de los tipos, expresiones y agentes reproductores de la violencia, así como de los espacios dentro del recinto donde más se reproduce.

\section{Tipos y expresiones}

De los siete tipos de violencia que define la Ley Especial Integral para una Vida Libre de Violencia para las Mujeres, seis se reproducen al interior de la universidad mediante diversas expresiones.

\section{Gráfico 1. Tipo de violencia más frecuente}

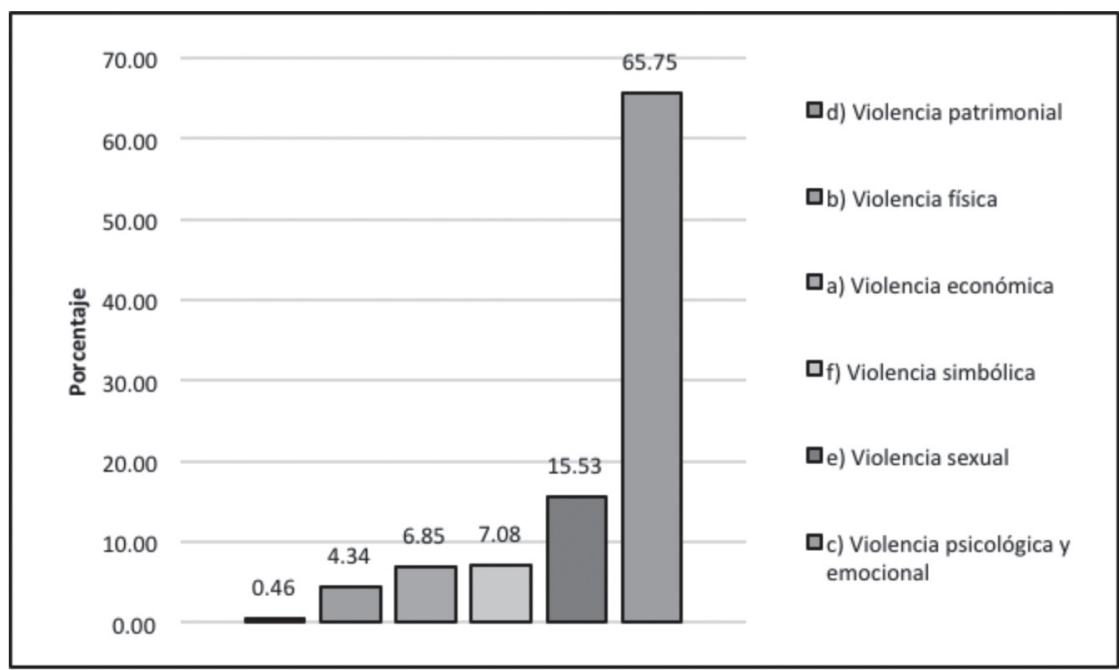

Fuente: elaboración propia.

Gráfico 2. Violencia económica

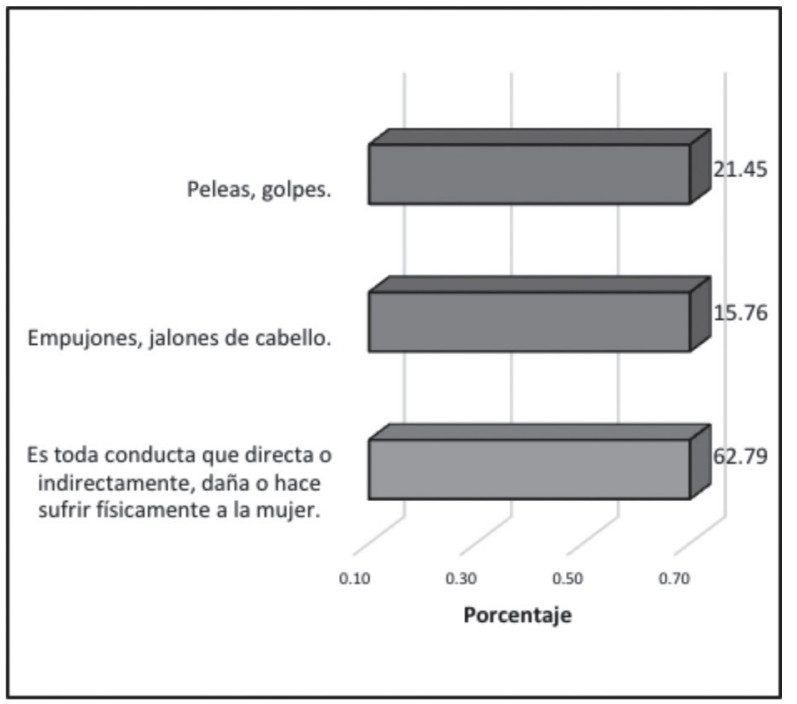

Gráfico 3. Violencia física

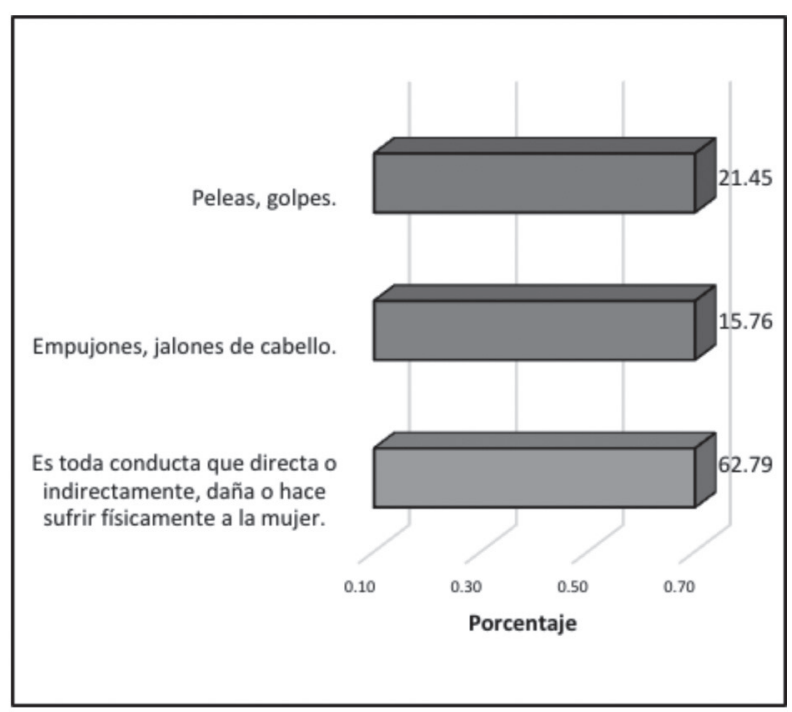

Fuente: elaboración propia. 


\section{Gráfico 4. Violencia psicológica y emocional}

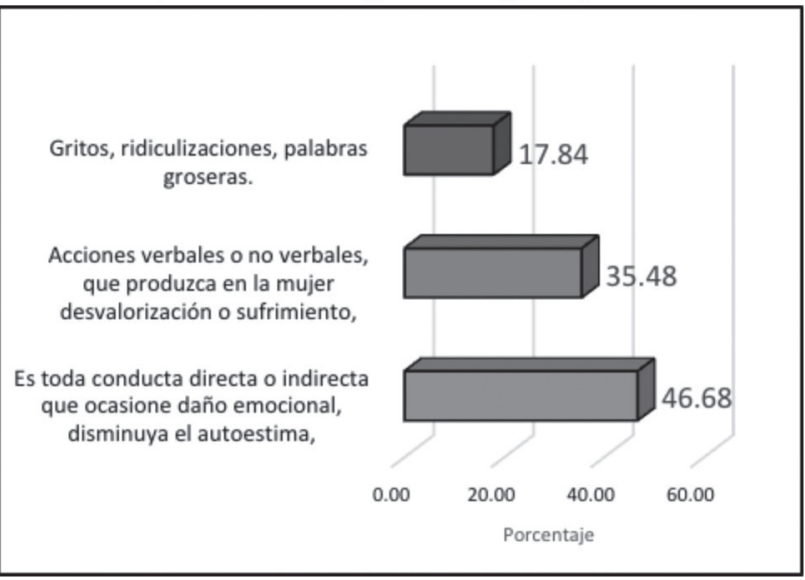

Fuente: elaboración propia.

\section{Gráfico 6. Violencia sexual}

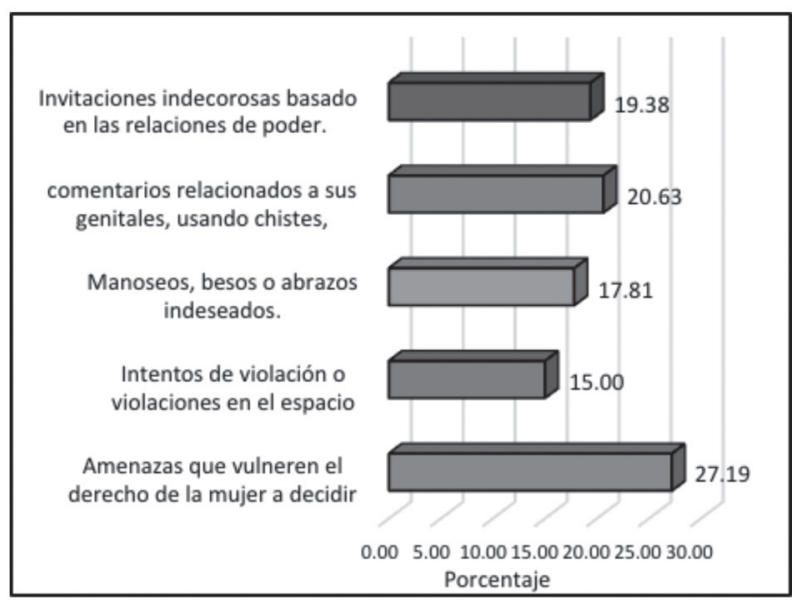

Fuente: Elaboración propia

\section{Gráfico 5. Violencia patrimonial}

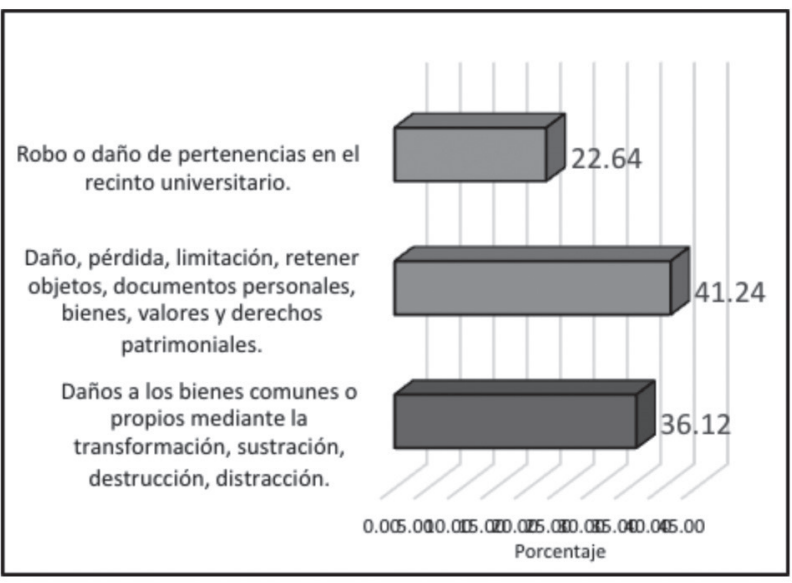

Gráfico 7. Violencia simbólica

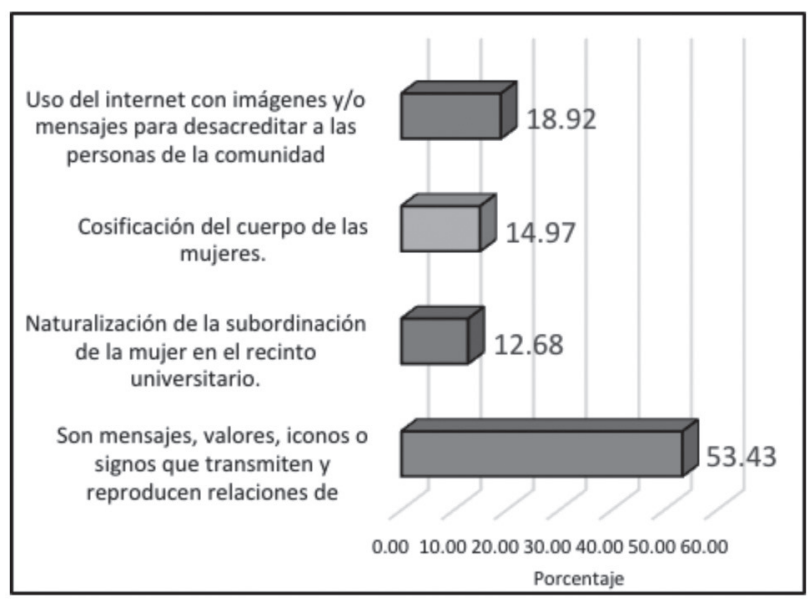




\section{Gráfico 8. Frecuencia de observación de violencia de género}

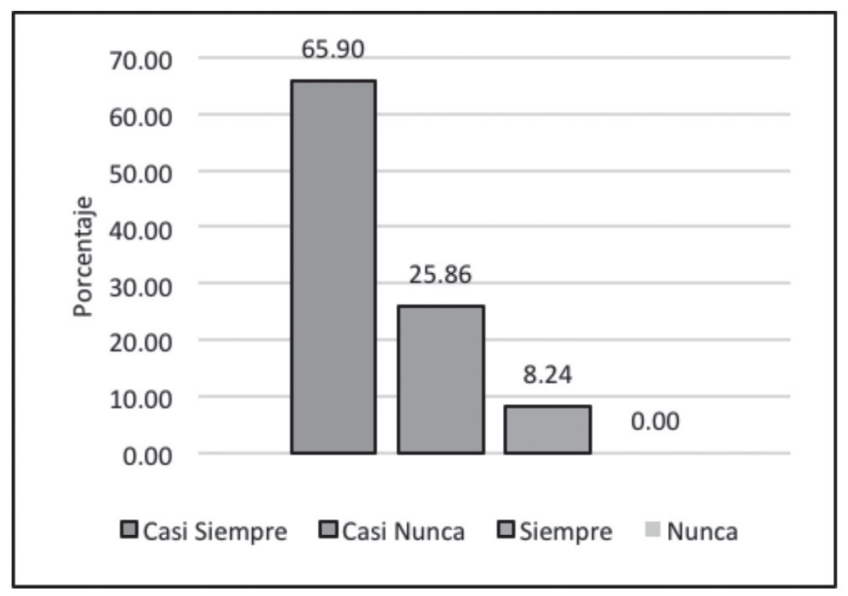

Fuente: Elaboración propia

II. Espacios de expresiones de violencia en los recintos universitarios

Tabla 3. Condiciones del ambiente universitario que contribuyen a la reproducción de la violencia

\begin{tabular}{|l|c|}
\hline Condición & $\%$ \\
\hline Indiferencia de la comunidad universitaria & 30,72 \\
\hline Espacios solitarios & 19,88 \\
\hline Desempeño del personal de seguridad universitaria & 18,09 \\
\hline Falta de iluminación & 12,72 \\
\hline Accesibilidad a los lugares & 9,64 \\
\hline Horarios & 8,35 \\
\hline Otros & 0,60 \\
\hline Total & 100,00 \\
\hline
\end{tabular}

Fuente: base de datos de encuesta.

La consulta revela que hay condiciones en el ambiente que permiten la reproducción de la violencia; y son las aulas, cubículos del personal docente y las oficinas los espacios en los que se observa mayor violencia. 
Gráfico 9. Espacio más frecuente donde se reproduce la violencia de género

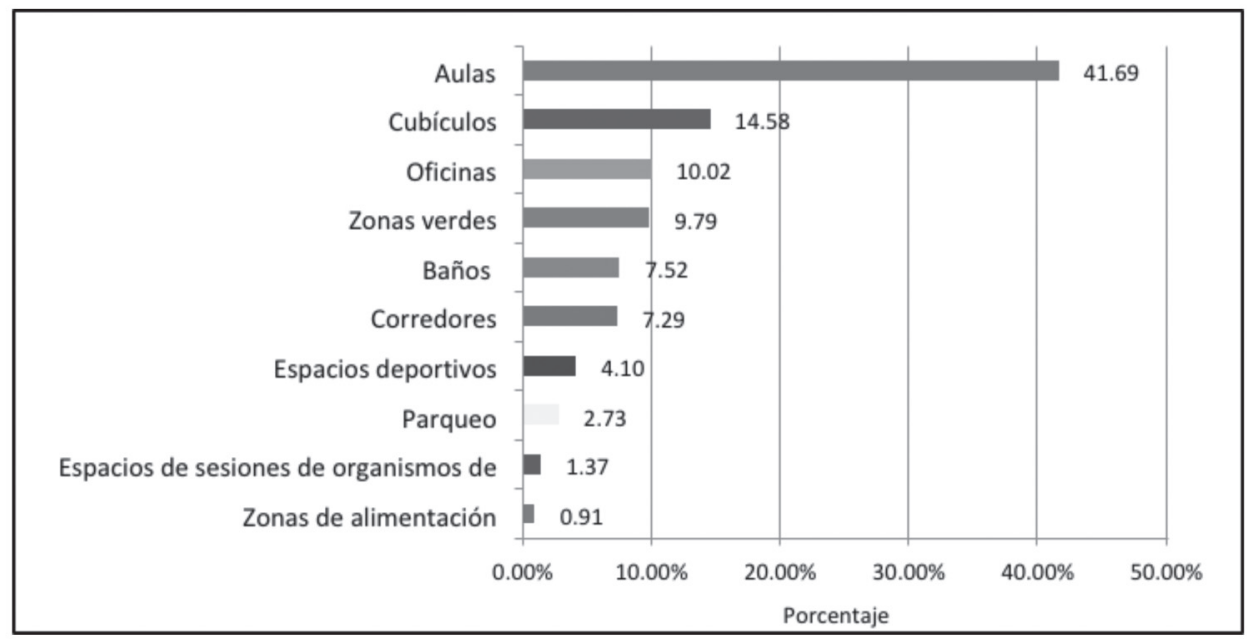

Fuente: elaboración propia.

\section{Agentes reproductores}

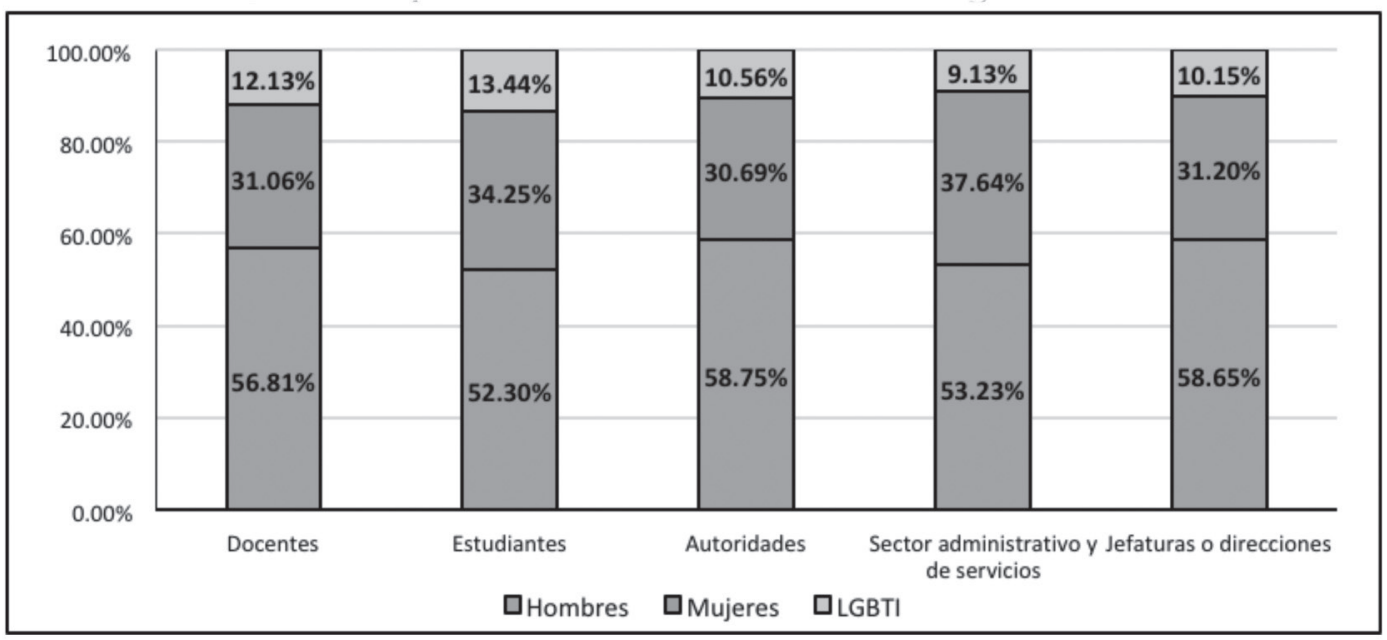

Fuente: elaboración propia. 
Gráfico 11. Aspectos negativos de las instancias responsables de atender problemas de violencia

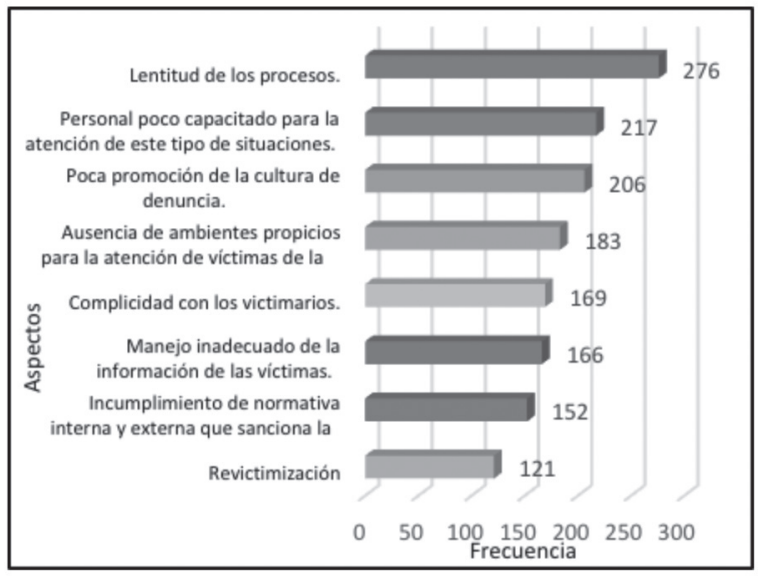

\section{Gráfico 12. Deficiencias en los procesos para atención de violencia}

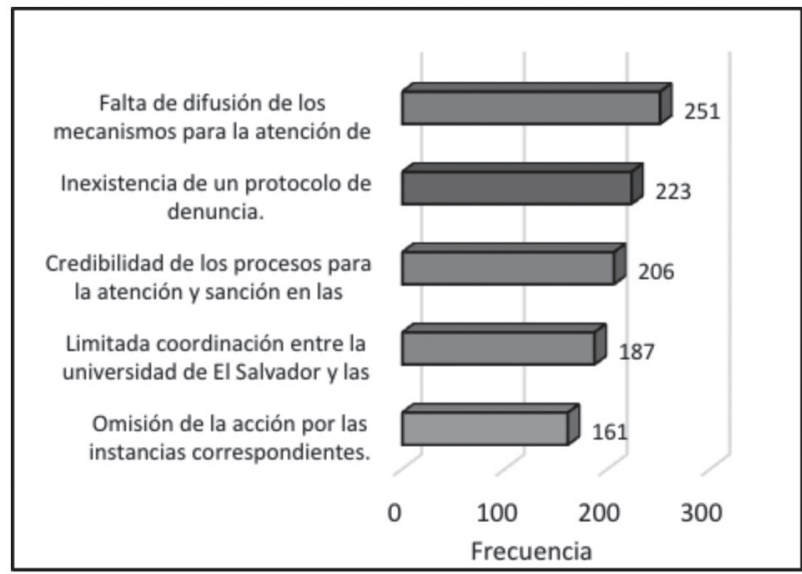

Fuente: elaboración propia.

IV. Percepción sobre la atención y reducción de la violencia en la Universidad de El Salvador

\section{Gráfico 13. Aspectos a mejorar en los procesos}

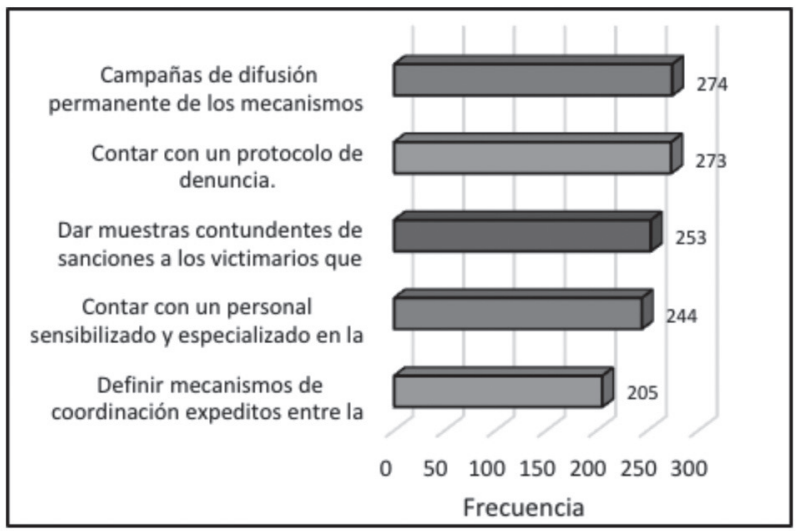

Gráfico 14. Encargados de impulsar mejoras

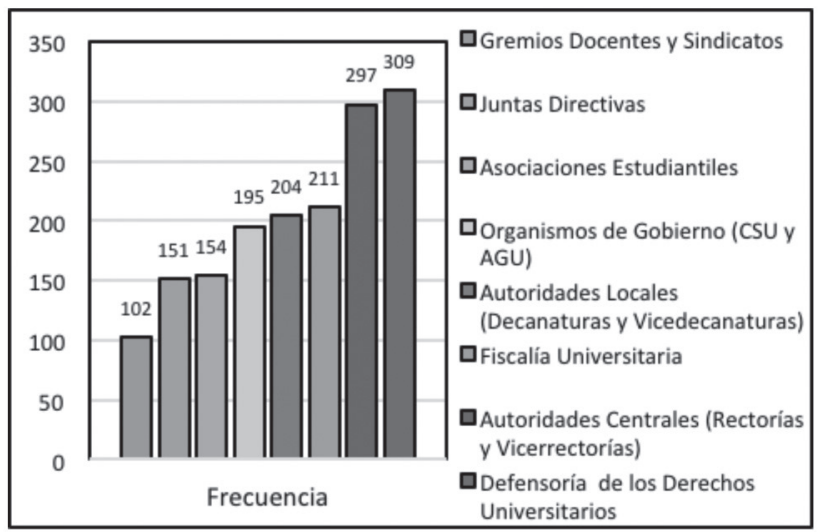

Fuente: elaboración propia. 


\section{Discusión}

La universidad no escapa a la reproducción de la violencia de género, explicada por la existencia de relaciones de poder derivadas de la prevalencia del sistema heteropatriarcal, entendido este como un "sistema de relaciones sociales caracterizado por la supremacía de lo masculino y la heterosexualidad, lo que genera mecanismos de discriminación por razón de sexo y orientación sexual hacia mujeres y personas Lesbianas, Gay, Transexuales, Bisexuales e Intersexuales LGBTI" (Verne, 2016). Otra razón de esta violencia es el comportamiento machista, definido como "la actitud de prepotencia de los hombres respecto de las mujeres" (RAE, 2018).

Los resultados de la consulta ponen en evidencia que son las mujeres las que sufren más violencia, alcanzando una frecuencia de respuestas del 64,82\%; para el caso de los hombres y personas LGBTI, los porcentajes son menores $(34,66$ y $18,53 \%$, respectivamente). La violencia obedece a la existencia de relaciones de poder entre hombres y mujeres, y entre sectores; sobre todo las que se expresan entre el personal docente y el sector estudiantil, así como entre quienes ostentan cargos de dirección o jefaturas y su personal subalterno.

Tipos de violencia de género. Analizar la violencia de género pasa por tomar en cuenta los diversos tipos y expresiones planteados en la Ley Especial Integral para una Vida Libre de Violencia contra las Mujeres. ${ }^{5}$ De acuerdo con esta Ley, los tipos de violencia son siete: económica, feminicida, física, psicológica y emocional, patrimonial, sexual y simbólica (Asamble Legislativa, 2011).

Según los resultados, 9 de cada 10 personas consultadas consideran que se reproduce algún tipo de violencia de género en la UES. De manera precisa, se identifican seis tipos dentro del recinto universitario, pero los tipos que más se reproducen son la violencia psicológica y emocional $(33,70 \%)$ y la sexual $(20,31 \%)$, mientras que los tipos de violencia menos identificados son la física $(11,43 \%)$ y la patrimonial $(6,06 \%)$.

Al profundizar sobre la forma en que se expresa la violencia psicológica y emocional, del total de personas encuestadas, un $46,68 \%$ afirma que se expresa como "toda conducta directa o indirecta que ocasione daño emocional, disminuya la autoestima, perjudique o perturbe el sano desarrollo de la mujer y hostigamiento". Una parte significativa de la población $(35,48 \%$ ) dijo que este tipo de violencia se da mediante "acciones verbales o no verbales, que produzcan en la mujer desvalorización o sufrimiento, mediante amenazas, exigencia de obediencia, coerción, culpabilización o limitaciones de su ámbito de libertad".

Sigue destacando como causal de este tipo de violencia, según las personas informantes, las desiguales relaciones de poder entre hombres y mujeres en todas las áreas de la universidad. Otro factor señalado es la orientación sexual, ya que se violentan con alguna frecuencia los derechos de las personas LGBTI. Las personas del sector administrativo consultado manifestaron sufrir violencia psicológica y emocional provocada por burlas de sus compañeras de trabajo, también discriminación por su condición física (por ser personas de baja estatura y con sobrepeso, por mencionar algunas razones). La tónica es la misma: el estudiantado que participó en los talleres identificó amenazas hacia las estudiantes y mujeres administrativas de parte de los agresores, para que desistan de hacer una denuncia formal ante los órganos competentes al interior de la universidad.

La violencia sexual fue identificada como la segunda modalidad más reproducida en el campus. Un $27,19 \%$ de la población consideró que esta se expresa con "amenazas que vulneran el derecho de la mujer a decidir voluntariamente su vida sexual", mientras que un $20,63 \%$ reconoce su manifestación mediante "comentarios relacionados a sus genitales, usando chistes, piropos, bromas de mal gusto"; un $19,38 \%$ opina que se da por "invitaciones indecorosas basadas en relaciones de poder", siendo las más afectadas las estudiantes de parte de docentes y los estudiantes. Algunas personas señalan haber recibido manoseos, tocamientos, así como besos y abrazos indeseados. Inclusive señalaron acontecimientos relacionados con el intento de violación de chicas en el recinto.

Según la percepción de la representación de autoridades y estudiantil entrevistada se reafirma que la violencia sexual es el segundo tipo de violencia más común en la UES, por su parte, el sector administrativo la identificó en primer lugar. De acuerdo a la consulta, los agentes 
reproductores son alumnos y vigilantes del género masculino, quienes ejercen violencia hacia las mujeres, así como los jefes hacia las trabajadoras. Se enfatiza en que este tipo de violencia es mayormente ejercida por los docentes hacia las estudiantes, quienes valiéndose de la relación de poder hacen ofrecimientos de notas a cambio de favores sexuales. "Lamentablemente no se cuenta con estadísticas, pero es muy conocida la paternidad de muchos docentes, que han embarazado a estudiantes" (opinión de una informante).

Preocupante es encontrar que 13 de cada 100 personas de la comunidad universitaria consultadas expresaron haber sido víctimas de violencia sexual en algún momento dentro del campus, siendo las mujeres en primer lugar las más afectadas por los docentes.

De las 656 personas de la muestra, el 53,43\% respondió que la violencia simbólica se expresa dentro de la universidad mediante "mensajes, valores, iconos o signos que transmiten y reproducen relaciones de dominación, desigualdad y discriminación en las relaciones sociales que se establecen entre las personas, por ejemplo, en el aula, las oficinas, espacios públicos". Asimismo, se da por medio de las redes sociales, carteles y mensajes que desacreditan, ridiculizan o minimizan principalmente a las mujeres. En el ambiente universitario persiste el lenguaje sexista y la cosificación de la mujer.

La violencia menos identificada es la patrimonial. Del total de personas encuestadas, un 41,24\% opinó que esta se expresa mediante "daño, pérdida, limitación, retener objetos, documentos personales, bienes, valores y derechos patrimoniales"; mientras que para un $36,12 \%$, por "daños a los bienes comunes o propios mediante la transformación, sustracción, destrucción, distracción"; esto ocurre mucho entre estudiantes.

Expresiones de violencia de género. Un 51,12 \% considera que la violencia económica se expresa mediante "actos encaminados a limitar, controlar o impedir el ingreso de las personas a puestos de trabajo, actividades formativas o de capacitación, particularmente de las mujeres" no solo cuando la jefatura es ejercida por un hombre, sino también cuando la ostenta una mujer. Otra modalidad de expresión con relativa importancia es la que se da cuando se genera retraso en la reclasificación o promoción laboral, sobre todo para las mujeres, a quienes se les exige una demostración mayor de sus competencias o habilidades respecto a los hombres (24,32\%); también suele ocurrir una prolongación en los períodos de pago cuando el personal trabaja tiempo adicional, poniendo en riesgo el cumplimiento de sus compromisos financieros y la satisfacción de las necesidades propias y de sus familias $(24,57 \%)$.

Respecto a las expresiones de violencia física, un 62,79\% manifestó que esta es "toda conducta que directa 0 indirectamente daña o hace sufrir físicamente a la mujer". Los datos de la encuesta coinciden con lo expresado por los estudiantes durante los talleres. Este tipo de violencia se visibiliza más en el maltrato ejercido por los hombres a sus parejas, lo que es muy común de ver en el recinto universitario, sobre todo en el sector estudiantil. Pese a ser un ambiente académico, se producen conatos de peleas, llegando a golpes y empujones no solo entre hombres sino también hacia las mujeres.

Espacios de expresiones de violencia en los recintos universitarios. Identificar los espacios donde se reproduce la violencia es de suma importancia para conocer las condiciones en las que se da, así como las que favorecen esa situación, de tal manera que las personas tomadoras de decisiones puedan impulsar acciones que contribuyan a mejorar el ambiente universitario.

La reproducción de la violencia en los diferentes espacios se ve potenciada por factores que pueden controlarse, tal es el caso de la falta de vigilancia y el desempeño mismo del personal de seguridad, la poca iluminación dentro del campus, sobre todo en las zonas más solitarias; la indiferencia de la comunidad universitaria, los horarios de algunas facultades, entre otros, favoreciendo a las personas agresoras.

Otros espacios en los que se reproduce con frecuencia la violencia son todas aquellas zonas donde existe un libre ingreso para cualquier persona que forma parte de la comunidad universitaria o que es externa a ella; entre estas están las zonas de alimentación, zonas verdes, plazas universitarias, de parqueos, los corredores y los espacios deportivos.

Agentes reproductores de violencia. Más del $55 \%$ de la población opina que son los hombres (en todos los sectores) quienes reproducen la violencia dentro de la UES, mientras que un $32,97 \%$ considera que son las mujeres. 
Una menor proporción respondieron que son las personas de la comunidad LGBTI.

Al analizar los datos recopilados de manera desagregada en relación con el género, se puede apreciar que el 31,57 \% opina que son los hombres del sector docente los que más reproducen la violencia; un $25,92 \%$ opina que son los estudiantes; y un 15,96 \% expresó que la violencia la reproducen los hombres que tienen cargos de autoridad (entiéndase en rectoría, vicerrectorías, decanaturas, representantes de gobierno central y local), seguidos de quienes ostentan alguna dirección o jefatura.

Dentro de las acciones de promoción y defensa de los derechos humanos de las mujeres universitarias, contemplados en la Política de Equidad de Género de la UES, se menciona "que la comunidad universitaria asuma responsablemente la seguridad de todas las personas, haciendo énfasis en la integridad personal (física, psicológica y sexual) de las mujeres, teniendo en cuenta los casos de violación que se dan al interior de la Ciudad Universitaria" (CEG, 2007), acción que no se está cumpliendo en su totalidad por la indiferencia de las personas y el miedo a represalias por parte de los agresores.

Según la LEIV, los tipos y modalidades de violencia se originan por relaciones desiguales de poder caracterizadas por la asimetría, el dominio y el control de una o varias personas sobre otra u otras, o por relaciones de confianza que se basan en los supuestos de lealtad, credibilidad, honestidad y seguridad que se establecen entre dos o más personas, donde la mujer se encuentra en posición de desventaja (Asamble Legislativa, 2011).

Pese al avance en el tema de derechos de las mujeres, al menos en lo formal, no se logra erradicar la violencia de género, donde las mujeres resultan ser las más afectadas. Ofensas, abusos, discriminación, burlas, acoso, impunidad de los agresores y el silencio de las mujeres agredidas son escenas cotidianas dentro del recinto universitario, sin que hasta el momento haya acciones concertadas entre las distintas instancias de la universidad para detenerlas.

\section{Propuesta para la atención y reducción de la violencia en la UES}

El reto que enfrenta la UES para erradicar la violencia de género no es una tarea fácil, principalmente porque más del $84 \%$ de las personas consideran que no existe un buen proceso de atención a las denuncias en las unidades universitarias encargadas de atender las denuncias; 8 de cada 10 personas no conoce la existencia de normativas que regulan la violencia de género en la institución; un $65 \%$ no conoce las instancias encargadas de aplicar la normativa universitaria.

En cuanto a las deficiencias que existen en los procesos, se confirma que no hay conocimiento de los mecanismos para la atención de denuncias; y no se cuenta con un protocolo para tal fin, ya que si bien el Reglamento Disciplinario define un procedimiento administrativo (DDU, 2017), no es suficiente para dar atención a los casos de violencia de género. Además, el procedimiento no está actualizado de acuerdo con la normativa nacional vigente sobre los derechos de las mujeres. Esto provoca que pocas personas quieran denunciar y lo hagan en las unidades aplicadoras de justicia de la universidad, llegando en algunos casos a que la persona violentada recurra a instancias fuera de la institución.

La investigación ha permitido no solo poner en evidencia una realidad que ya se percibía, sino también disponer de información cuantitativa a partir de la cual se vuelve necesaria la toma de decisiones. Por tal razón, se buscó conocer la opinión de las personas informantes sobre las acciones que se deben implementar para contrarrestar la violencia de género. Se listan a continuación las principales propuestas que más se repiten, tanto en la encuesta como en desarrollo de talleres, grupos focales y entrevistas. No están precisadas en ningún orden, pero hay algunas que son de aplicación inmediata en tanto que otras requieren de más discusión y recursos para su implementación.

1. Campañas de difusión sobre los derechos de la población estudiantil y para dar a conocer las instancias encargadas de atender denuncias.

2. Talleres y charlas sobre los derechos de las mujeres y la violencia de género.

3. Crear protocolos de denuncia armonizados con la normativa nacional vigente.

4. Fortalecer el Centro de Estudios de Género, asignando presupuesto, infraestructura y personal para que cumpla su papel de ente rector en el tema de género dentro de la UES. Esta instancia debe dar seguimiento a todas las denuncias a fin de garantizar que se aplique la normativa interna y nacional. 
5. Sensibilización, capacitación y profesionalización a todo el personal encargado de atender las denuncias dentro de la UES, es decir la Asamblea General Universitaria (AGU), Consejo Superior Universitario (CSU), Defensoría de los Derechos Universitarios (DDU), Rectoría y vicerrectorías, Juntas Directivas y la Fiscalía, entre otras.

6. Armonización de toda la legislación universitaria con las leyes nacionales.

7. Crear unidades especializadas para la atención de la violencia de género dentro de la UES en todos sus campus.

8. Actualizar la Política de Equidad de Género de la UES y su Plan de Igualdad, para la correspondiente ratificación por la AGU.

9. Sancionar a quienes incurran en violencia de género independientemente del cargo que ostente, evitar la complicidad, impunidad y lentitud de los procesos.

10. Aprovechar los convenios que se han firmado con organismos nacionales e internacionales con el fin de mejorar esfuerzos de prevención y erradicación de la violencia de género.

11. Crear una escuela de formación permanente sobre legislación universitaria, nacional e internacional, derechos humanos e igualdad.

12. Reformar el reglamento de la DDU, de manera que sea un órgano vigilante de cualquier tipo de violación de los derechos universitarios, no solo en aquellos donde prevalezca una relación de poder.

13. Dar cumplimiento al artículo 23 de la LIE para promover la participación de más mujeres en organismos de toma de decisiones.

Según la opinión de las personas informantes, las instancias que se encargan de velar por los derechos dentro de la UES deben transversalizar el enfoque de género y de derechos humanos, al mismo tiempo que se impulsen acciones afirmativas principalmente hacia las mujeres. Para quienes la comunidad les lanza el desafío de estar al tanto de los avances en el tema de derechos para prevenir y sancionar la reproducción de la violencia, estos son los siguientes:

1. Defensoría de los Derechos Universitarios: sus funciones están enmarcadas en la protección, defensa o tutela de los derechos universitarios contra actos u omisiones de autoridades que presumiblemente amenacen lesionar o lesionen los derechos o facultades de los miembros, y la promoción y difusión de esos derechos (UES, 2018).

2. Autoridades centrales: la Rectoría es la máxima unidad ejecutiva de la universidad y tiene a su cargo la representación legal de la institución. Ejecuta y hace cumplir las resoluciones de la AGU y del CSU (UES, 2018).

3. Fiscalía: ejercerá sus atribuciones y deberes con independencia de los demás órganos y funcionarios, velará por el estricto cumplimiento de la Ley Orgánica, de los reglamentos y de las normas válidamente emanadas de los órganos competentes que integran el gobierno universitario, así como de las demás leyes que fueren aplicables a las actividades de la universidad (UES, 2018).

Existen otras instancias que juegan un papel clave en impulsar medidas para la igualdad y la erradicación de la violencia, es el caso de los organismos de gobierno central, autoridades locales y juntas directivas, que son los encargados de los ámbitos disciplinarios, así como las asociaciones estudiantiles y gremios de docentes y sindicatos, para que se vuelvan garantes del respeto a los derechos de las personas que integran de la comunidad universitaria.

Es interesante la petición casi generalizada de parte de todos los sectores consultados, respecto a la necesidad urgente de crear una unidad especializada e independiente de las facultades y otras instancias que conforman la UES, que se encargue de recibir y canalizar las investigaciones relacionadas con casos de denuncias por violencia de género, que dé respuesta inmediata a las víctimas, a fin de garantizar autonomía e independencia en los procesos, para evitar la complicidad e impunidad con los agresores.

La comunidad universitaria considera que son muchos los retos que deben superarse para mejorar la situación que se vive en cuanto a reproducción de la violencia de género, ya que la UES es un reflejo de la sociedad salvadoreña, donde por ahora no existe un ambiente de respeto de los derechos humanos, sobre todo para las mujeres.

\section{Conclusiones}

- El Salvador ha logrado avances significativos en cuanto a la legislación que defiende los derechos de las mujeres, así como de otros sectores de la sociedad 
históricamente vulnerados. De igual forma ha firmado y ratificado diferentes acuerdos internacionales; eso dota de un marco jurídico suficiente para apostar a una sociedad más igualitaria, justa e incluyente.

- $\quad$ La UES es una institución que no escapa a los sistemas heteropatriarcal y machista. La evidencia encontrada afirma que se reproduce la violencia de género, siendo las mujeres en los diferentes estratos de la comunidad universitaria las más afectadas.

- La existencia de relaciones de poder en el aula universitaria y en las oficinas, el desconocimiento de los derechos y la indiferencia de la misma comunidad universitaria son las principales causas de la violencia. Docentes, personal de servicios, autoridades y jefes aparecen como los principales agentes reproductores de la violencia, sin que haya mayor sanción a los agresores.

- Los espacios donde más se identifica la reproducción de la violencia son las aulas universitarias, cubículos y oficinas, debido a la existencia de relaciones de poder y a la indiferencia de la misma comunidad universitaria.

- Pese a la vigencia de un marco normativo nacional y al interior de la UES sobre los derechos de las mujeres, no se tiene mayor conocimiento de este, tampoco de las instancias responsables de la implementación de la legislación al interior de la institución. Esta situación contribuye a la falta de denuncia, a la que se debe sumar el temor y la poca confianza en los procesos debido a la complicidad que suele presentarse, sobre todo entre los hombres.

- La reproducción de la violencia en sus diferentes expresiones en la UES es reconocida por todos los sectores consultados; sin embargo, es la violencia psicológica y emocional la que más se reproduce en todo el recinto universitario, ya que como en toda la sociedad se ha naturalizado, y afecta sobre todo a las mujeres.

- Se carece de una cultura de denuncia por falta de credibilidad en los procesos, debido a la complicidad y omisión en la aplicación de las sanciones a las personas agresoras, por lo que muchos hombres continúan acosando a pesar de ser señalado en silencio su comportamiento.
- La incorporación de la UES a la Red de Investigación en Diferenciales de Género en la Educación Superior de Iberoamérica representa una valiosa oportunidad para abordar temáticas como la estudiada en esta oportunidad, y otras que pongan en evidencia las brechas existentes entre los géneros en todos los sectores de la universidad. Además, permite la construcción de datos e indicadores que sirvan para el diseño e implementación de medida institucionales que favorezcan la igualdad y el respeto a los derechos de todos los sectores.

\section{Referencias}

Administración Académica, (2017). Estadísticas. Universidad de El Salvador. Obtenido de: https://academica.ues. edu.sv/estadisticas/poblacion_estudiantil.php

Asamblea Legislativa, d. I. (2011). "Ley Especial Integral para una Vida Libre de Violencia contra las Mujeres". San Salvador: Diario Oficial N. ${ }^{\circ} 2$, Tomo 390.

Asamblea General Universitaria, (2001). "Ley Orgánica de la Universidad de El Salvador". El Salvador: Diario Oficial $\mathrm{N}^{\circ} 113$, tomo 351.

Asamblea General Universitaria, (2001). "Reglamento Disciplianario de la Universidad de El Salvador". San Salvador: Diario Oficial 113, Tomo 351.

Asamblea Legislativa, R. d. (2011). "Ley de Igualdad, Equidad y Erradicación de la Discriminación contra las Mujeres". El Salvador: Diario Oficial, No.2, Tomo 390.

CEG, (2007). Política de Género. El Salvador: Universidad de El Salvador.

DDU, (2017). Legislación Universitaria. San Salvador: Imprenta Universitaria.

Diario El Mundo, (5 de junio de 2017). "700 empleados de la UES afectados por embargo de prestamistas".

RAE, (2018). Diccionarios de lengua española. Obtenido de http://dle.rae.es/?id=QlqTEX0|Qlr66uc|Qltkqeu

Red, M. e. (2008). ¿Qué es el Patriarcado? Editorial Biblos.

UES, U. d. (2018). "Universidad de El Salvador, UES". Recuperado el 25 de julio de 2018 de https://www.ues. edu.sv/administracion

Coteues, (2007) unidad técnica de evaluación. Universidad de El Salvador. obtenido de http://coteues.ues.edu.sv/

Verne (17 de 6 de 2016). "Qué quiere decir Alberto Garzón cuando habla de heteropatriarcado". 